\title{
CARACTERIZACIÓN DE LA POBREZA OCULTA Y SU EFECTO EN LA SOSTENIBILIDAD ECONÓMICA DE LAS PEQUEÑAS Y MEDIANAS EMPRESAS DE LA LOCALIDAD DE USAQUÉN (BOGOTÁ - COLOMBIA)*
}

\author{
MARTHA RUTH MENDOZA TORRES S** $^{* *}$ ORLANDO TARAZONA MORALES $S^{* * *} \&$ LUCERO DUQUE VARGAS $S^{* * * * *}$ \\ UNIVERSIDAD EL BOSQUE (BOGOTÁ)
}

Recibido/ Received/ Recebido: 04-11-2011 - Aceptado/ Accepted / Aprovado: 29-12-2011

\begin{abstract}
Resumen
El propósito de este artículo es mostrar los resultados de la investigación realizada en la localidad de Usaquén, la cual caracterizó la pobreza oculta o empobrecimiento de los hogares de clases media y alta, fenómeno que ha generado cambios en hábitos de compra y consumo, produciendo diferentes tipos de impacto en la sostenibilidad económica de las pequeñas empresas del sector. Para esto se seleccionaron dos poblaciones de estudio en la localidad: hogares de estratos 3 , 4 y 5 y empresas pequeñas y medianas. Como metodología se utilizó la triangulación propuesta por Okuda \& Gómez (2005), consistente en la aplicación de métodos cualitativos, entrevistas y grupos focales, y métodos cuantitativos, muestreo por conglomerados para aplicación de encuestas, que fueron procesadas mediante SPSS ${ }^{\circledR}$ Versión 14. Se encontró que efectivamente los hogares en estado de pobreza oculta han tenido cambios en sus hábitos de compra, ante los cuales las empresas han desarrollado estrategias adaptativas para preservar sus utilidades, como el desarrollo de nuevos servicios y el aumento del surtido de productos, para responder a los cambios de la demanda derivados de este tipo de pobreza, en aras de garantizar su sostenibilidad económica.
\end{abstract}

Palabras clave: Pobreza oculta, Pequeñas y medianas empresas, Sostenibilidad económica.

Artículo científico que presenta los resultados finales del proyecto de investigación PCI 2009-46 "Impacto de la pobreza oculta en la sostenibilidad económica de las Mipymes de la localidad de Usaquén”, financiado por la Universidad El Bosque, Facultad de Ingeniería Industrial, Grupo de investigación en Innovación tecnológica y productividad GINTECPRO, desarrollado por profesores de la Facultad durante el año 2010.

** Ingeniero Industrial Universidad de los Andes, Magíster en Ingeniería Industrial Universidad de los Andes, docente investigador Facultad de Ingeniería Industrial Universidad El Bosque, grupo de investigación GINTECPRO. Correo electrónico: mendozamartha@ unbosque.edu.co

**** Ingeniero Industrial Universidad Antonio Nariño, Magíster en Administración de Empresas Universidad Nacional de Colombia, docente investigador Facultad de Ingeniería Industrial Universidad El Bosque. Correo electrónico: otarazona@unbosque.edu.co

***** Economista Universidad de Caldas, Especialista en Gestión para el Desarrollo Empresarial Universidad de Santo Tomás, docente investigadora Facultad de Ingeniería Industrial Universidad El Bosque. Correo electrónico: luceroduque@hotmail.com 


\title{
CHARACTERIZATION OF HIDDEN POVERTY AND THEIR EFFECT ON ECONOMIC SUSTAINABILITY OF SMALL AND MEDIUM COMPANIES OF USAQUÉN LOCALITY (BOGOTÁ - COLOMBIA)
}

\begin{abstract}
The purpose of this article is to show the results of a research made in Usaquén Locality, in which hidden poverty or household impoverishment of medium and high class was characterized, this phenomenon has generated changes in buying and consumption, habits producing different types of impact on economic sustainability of sector's small companies. In this situation, companies have developed adaptive strategies to preserve their income and benefit such as: new services development, complementation of product portfolio and serve to different client profiles, in order to achieve responding to changing demand requirements of goods and services, in order to guarantee their economic sustainability. For such effect, the triangulation proposed by Okuda \& Gómez (2005) was used, which combines qualitative and quantitative methods.

Key words: Hidden poverty, Socioeconomic status, Small and medium companies, Economic sustainability.

\section{CARACTERIZAÇÃO DA POBREZA OCULTA E SEU EFEITO NA SUSTENTABILIDADE ECONÔMICA DAS PEQUENAS E MÉDIAS EMPRESAS DA LOCALIDADE DE USAQUÉN (BOGOTÁ - COLÔMBIA)}

\begin{abstract}
Resumo
O propósito deste artigo é mostrar os resultados da pesquisa realizada na localidade de Usaquén, que caracterizou a pobreza oculta ou empobrecimento dos lares de classes média e alta, fenômeno que gerou mudanças em hábitos de compra e de consumo, produzindo diferentes tipos de impacto na sustentabilidade econômica das pequenas empresas da localidade. Frente a esta situação, as empresas desenvolveram estratégias de adaptação para preservar suas receitas e utilidades tais como: o desenvolvimento de novos serviços, complemento da lista de produtos e atenção a diferentes perfis de clientes, conseguindo assim responder às necessidades da demanda mutante de bens e serviços, em função de garantir sua sustentabilidade econômica. Empregou-se a triangulação proposta por Okuda e Gómez (2005), a qual combina métodos qualitativos e quantitativos.
\end{abstract}

Palavras chave: Pobreza oculta, Pequenas e médias empresas, Sustentabilidade econômica.

Mendoza, M. Tarazona O. \& Duque, L. (2011). Caracterización de la pobreza oculta y su efecto en la sostenibilidad económica de las pequeñas y medianas empresas de la localidad de Usaquén (Bogotá - Colombia). En: Revista de la Facultad de Ciencias Económicas de la Universidad Militar Nueva Granada. rev.fac.cienc.econ, XIX, (2)

JEL: I32, L25.

\section{Introducción}

Esta investigación surgió como respuesta a la inquietud manifestada por la Alcaldía Local de Usaquén, respecto al desconocimiento del fenómeno de po- breza oculta en las clases medias y altas de la localidad, y como éste puede estar generando además de retos sociales, retos económicos que requieren ser atendidos con estrategias acordes a las características de los mismos. 
Este artículo presenta los resultados de esa investigación realizada en la Facultad de Ingeniería Industrial de la Universidad El Bosque, Bogotá (Colombia), cuyo objetivo fue caracterizar la pobreza oculta en Usaquén, identificar cambios en la composición y volumen de demanda de bienes y servicios derivados de este fenómeno e identificar patrones de relación entre esos cambios y la sostenibilidad económica de las pequeñas empresas de esta localidad.

Se presenta una revisión teórica sobre que es la pobreza y más exactamente la pobreza oculta como fenómeno propio de las clases medias y altas. Luego hacemos una presentación de las características que definen lo que es una pequeña empresa en Colombia, continuando con una reflexión sobre la importancia de desarrollar la flexibilidad y capacidad de gestión de las mismas para adaptar su oferta de bienes y servicios a los cambios de la demanda, y particularmente, aquellos derivados de la pobreza oculta, que al no ser explícita, exige mayor monitoreo de las necesidades de sus clientes. A continuación, se presenta la metodología empleada en la realización de la investigación cualitativa, exploratoria y descriptiva aplicada a dos poblaciones de estudio, hogares y empresas, la cual hizo uso de la triangulación consistente en la aplicación de métodos cualitativos (entrevistas a empresarios y grupos focales con la participación de jefes de hogar) y métodos cuantitativos (muestreo por conglomerados para aplicación de encuestas a empresarios y hogares) permitiendo cruzar los resultados cualitativos y cuantitativos, minimizando así el sesgo y aumentando la consistencia $\mathrm{y}$ validez de los hallazgos.

Finalmente, se presentan los resultados los cuales muestran reducción en los ingresos de los hogares estudiados derivados de pérdida del empleo de uno de los integrantes de la familia, cambio a un empleo de menor remuneración o reducción de las ventas de la empresa propia, lo que conlleva a cambios en los hábitos de compra y consumo de los hogares estudiados, siendo los más relevantes el cambio de sitio de compra de bienes y servicios en busca de menores precios y la reducción de la compra de determinados bienes y servicios. En cuanto el estudio de empresas, este confirma los cambios de hábitos de compra de los hogares, añadiendo el hecho de que estas han desarrollado estrategias adaptativas para responder a los cambios del volumen y composición de demanda de bienes y servicios, manifiestas en el desarrollo de nuevos servicios y aumento de surtido, y a la aparición de nuevos clientes provenientes de estratos diferentes a los que habitualmente atienden.

\section{Pobreza y pobreza oculta}

Ante la idea de pobreza oculta cabe preguntarse ¿Qué es la pobreza oculta? ¿Cómo se afecta la satisfacción de las necesidades de la población que sufre pobreza oculta? ¿Cómo cambian los hábitos de compra y consumo por efecto de la pobreza oculta? ¿Cuáles son las causas de este tipo de pobreza?

Para dar respuesta a estos interrogantes es preciso abordar la definición de pobreza, aunque la primera dificultad es la variedad de conceptos y definiciones que existen dependiendo del país, región, cultura, tradición y percepción de cada individuo. Platón (citado por Sen, 1987) expresó que "la pobreza no viene por la disminución de las riquezas, sino por la multiplicación de los deseos," haciendo referencia a la percepción individual. Por otra parte, la sociedad de consumo de hoy en día, conduce a algunas personas a desear estar a la vanguardia de la moda, generando en ellas el afán por obtener estatus y aprobación social (Sen, 1987), lo que las conduce a percibirse a si mismas como pobres, si no pueden acceder a los bienes y servicios que representan el reconocimiento y aceptación de un grupo social determinado (Bauman, 2007, 116).

A su vez Ravallion, Shaohua, \& Prem (2007) argumentan que la pobreza existe en una sociedad, cuando una o más personas carecen de un nivel de bienestar económico que les permita tener un mínimo razonable frente a los estándares de esa sociedad. Es decir emerge la pobreza más por una percepción surgida de la comparación con esos estándares, que por una situación real de carencia de lo mínimo para subsistir.

Al respecto Dréze \& Sen (1989) comentan que "la pobreza no solamente debe mirarse como un dilema económico cuantitativo (ingresos bajos, carencia o 
insuficiencia de bienes materiales, entre otros)" sino que también "se deben incluir en su estudio elementos de corte cualitativo, como el acceso al desarrollo de capacidades y titularidades". Esto implica el buscar y aprovechar oportunidades de mejora de calidad de vida y acceder a grupos sociales que representen mayor información, conocimiento de la realidad y manejo mesurado de los recursos.

Existen diferentes visiones sobre la pobreza, por lo que una mirada a estas puede ayudar a comprender mejor las distintas aproximaciones a lo que esta es, apreciable en la tabla 1 . Pero como las necesidades y deseos humanos son fuerzas, que mueven al desarrollo de las personas (Maslow, 1985), si se satisfacen la persona pasará de estados básicos, relacionados con las necesidades fisiológicas y psicológicas, a estados superiores relacionados con el desarrollo personal y la autorrealización. Dependiendo del estado en que se encuentre la persona, surgirán nuevos deseos relacionados con el acceso a bienes y servicios, según los estándares del ámbito social en que se desenvuelve.

En esta escala, la incapacidad para satisfacer necesidades de supervivencia básica, es decir fisiológicas, corresponde a pobreza en términos absolutos (Pérez, 2004). Pero cuando se trata de la incapacidad de la persona para llevar un nivel de vida conforme al de la sociedad en la que vive, o lo que es lo mismo, de cubrir las necesidades que, sin estar relacionadas con la supervivencia, son consideradas normales o básicas en esa sociedad, se está ante una pobreza relativa (Pérez, 2004) o por comparación. En la misma línea de Pérez, en ambos casos, hay una dimensión subjetiva puesto que la definición de necesidades dependerá de lo que la persona perciba como tal.

Tabla 1. Definiciones de pobreza

\begin{tabular}{|c|c|c|}
\hline $\begin{array}{l}\text { CONCEPTO DE } \\
\text { POBREZA }\end{array}$ & CARACTERÍSTICAS & AUTORES \\
\hline $\begin{array}{l}\text { Ausencia de recursos/ } \\
\text { Privación }\end{array}$ & $\begin{array}{l}\text { Falta de un recurso ante un referente de dotación de recursos que posee un indivi- } \\
\text { duo, grupo o comunidad que presenta un grado de bienestar. Como consecuencia } \\
\text { de esta privación se presenta una pérdida de bienestar. } \\
\text { En el modelo neoclásico se asume equidad en la dotación inicial de un individuo o } \\
\text { familia, éste es un fuerte supuesto del modelo. }\end{array}$ & $\begin{array}{l}\text { Neoclásicos. } \\
\text { La situación de pobreza y desempleo es } \\
\text { derivada, según esta teoría del inadecuado } \\
\text { nivel de capital humano (Jiménez, 2007). } \\
\text { Visión: Materialista/utilitarista }\end{array}$ \\
\hline $\begin{array}{l}\text { Insuficiencia de medios } \\
\text { para adquirir recursos. }\end{array}$ & $\begin{array}{l}\text { Inequitativa distribución del ingreso que genera un obstáculo a los individuos para } \\
\text { acceder a recursos o falta de ingresos que genera brechas entre niveles de vida de } \\
\text { las personas. Nivel mínimo de vida razonable frente a los estándares de la sociedad. }\end{array}$ & $\begin{array}{l}\text { Autores: Atkinson \& Bourguignon (1987) } \\
\text { Visión: inequidad social/ consecuencialista }\end{array}$ \\
\hline $\begin{array}{l}\text { Necesidades Básicas } \\
\text { (privación). }\end{array}$ & $\begin{array}{l}\text { Mínimo de bienes que debe tener una persona o grupo para encontrarse en una si- } \\
\text { tuación digna de vida. Pérdida de bienestar por tal circunstancia. Existe quién puede } \\
\text { solucionar los problemas de necesidades con provisión de bienes públicos. }\end{array}$ & $\begin{array}{l}\text { Banco Mundial (2000-2001) } \\
\text { Visión: materialista/ procedimentalista }\end{array}$ \\
\hline $\begin{array}{l}\text { Problema de capacidades } \\
\text { individuales/ titularidades }\end{array}$ & $\begin{array}{l}\text { Se concentra en las capacidades de los individuos y en su posibilidad de desarrollar } \\
\text { habilidades, allí lo material pasa a un segundo plano (sin dejar de ser importante). } \\
\text { Las titularidades permiten analizar cómo las estructuras sociales le permiten a los } \\
\text { individuos acceder a un bien. Cualquier desigualdad en la estructura social genera } \\
\text { un problema. }\end{array}$ & $\begin{array}{l}\text { Dréze \& Sen. (1989). } \\
\text { Visión: individualismo/liberalismo }\end{array}$ \\
\hline Clase social/desigualdad & $\begin{array}{l}\text { La extracción social genera desigualdades entre grupos sociales, lo cual explica el } \\
\text { bajo nivel de bienestar. Hay apropiación de un grupo del excedente que otros pro- } \\
\text { ducen. Problemas de estructura social. Dificultad en acceso a bienes de posición. }\end{array}$ & $\begin{array}{l}\text { Escuela: Marxista. } \\
\text { Visión: explotación/socialismo. }\end{array}$ \\
\hline
\end{tabular}

1 Fuente: Serrano (1996). 
Una vez definidas la pobreza absoluta y relativa, emerge el concepto de pobreza oculta o nuevos pobres, referente a aquellas personas que, a pesar de disponer de empleo $\mathrm{u}$ otras fuentes de ingreso, no poseen los recursos suficientes para cubrir sus necesidades, las cuales incluyen las básicas y las referentes a los estándares de la sociedad. Tradicionalmente la pobreza se asocia con personas sin ingresos, pero se da el caso de quienes viven en algún estado de pobreza a pesar de contar con algún tipo de ingreso (Pérez, 2004).

Según Marpsat $(2006,65)$, la evidencia de la pobreza oculta no es visible en el espacio público, pues tiene manifestaciones más profundas, tal como lo muestra una investigación efectuada en Francia, donde se encontró que la pobreza afecta por ejemplo a personas que a pesar de ser propietarios del lugar donde habitan, reciben pensiones o rentas insuficientes para cubrir sus necesidades, o sufren de algún tipo de discapacidad física o sicológica, factores que los conducen a tener su vivienda en mal estado de mantenimiento y a recurrir a servicios sociales como comedores comunitarios o albergues para cubrir necesidades de alimentación y alojamiento.

Son estas diferencias en la manifestación de la pobreza, las que dificultan la medición de la misma. El economista Amartya Sen, premio nobel en 1998, en el estudio denominado "Poverty: An Ordinal Approach to Measurement" (Sen, 1976, 219-231) hizo la distinción entre dos problemas concretos al momento de medir la pobreza: El primero, "identificar a los pobres entre la población total”, lo cual “(...) implica la selección de una "línea de pobreza"", y el segundo, construir un "índice de pobreza usando la información disponible de los pobres".

Pero fue Rowntree $(2002,436)$ el primero que tuvo en cuenta los problemas que supone tratar de definir la pobreza, los cuales consideró en su enfoque basado en la línea de pobreza. Inicialmente planteó que una familia estaba en la pobreza si sus ingresos totales eran "insuficientes para obtener lo mínimo necesario para el mero mantenimiento de la eficiencia física". Pero después de llegar a esa definición descubrió que "encontrar un valor monetario para ese "mínimo necesario" de alimentos y de otros bie- nes era una tarea plagada de dificultades conceptuales y prácticas" (Ruiz, 2009, 67-100), ya que existe otra dimensión que añade complejidad al problema, consistente en la "representación de las relaciones sociales" que depende del contexto en que éstas se den (Guerrien, 1998), dando origen a la percepción de pobreza relativa.

Es a partir de esta percepción que los individuos efectúan intercambios para el consumo y la producción de bienes y servicios (Guerrien, 1998) dando origen al juego de oferta y demanda. La oferta es tipificada por la producción y la demanda por el consumo de individuos, familias y empresas. Y es en la interacción entre estas unidades económicas básicas que se origina la formación de precios, cantidades producidas $\mathrm{u}$ ofertadas $\mathrm{y}$ cantidades demandadas, siendo determinadas estas últimas por la evolución en los hábitos de compra y consumo de los individuos y familias, los que se relacionan tanto con sus niveles de ingresos, como con sus percepciones de los estándares que la sociedad entiende como indicadores de riqueza o pobreza.

En consecuencia, son los hábitos de compra y consumo de los individuos y las familias y su dinámica de cambio, los que determinan la estructura o composición de la demanda y sus volúmenes o tasas en un momento determinado, así como la oferta de productores y comercializadores, en el libre juego de oferta y demanda. Es así, como los productores y comercializadores se ven enfrentados al reto de sincronizar sus estructuras y volúmenes de oferta con las estructuras y volúmenes de una demanda dinámica por naturaleza, la cual depende de los niveles de riqueza o pobreza de individuos y familias, sea esta última absoluta o relativa, visible u oculta.

\section{Sincronización de la composición de la oferta y demanda}

Christopher \& Braithwaite (1989, 192-197) detectaron que, a pesar de que las empresas se esfuerzan por ofrecer a sus clientes "el producto correcto, en el tiempo correcto y en el lugar correcto" en la práctica se presenta una deficiente planeación entre mercadeo y manufactura, o entre mercadeo y compras lo 
que impide cumplir con esa oferta, por la desarticulación entre composición y disponibilidad de la oferta de las empresas y la composición y comportamiento de la demanda. A esto se suma la falta de sincronización entre volumen o tasa de demanda y volumen o tasa de abastecimiento de materias primas o de mercancías para la venta, lo que da como resultado demora en la respuesta a la demanda, y agregación de costos, antes que de valor en todo el ciclo ventas - producción-compras.

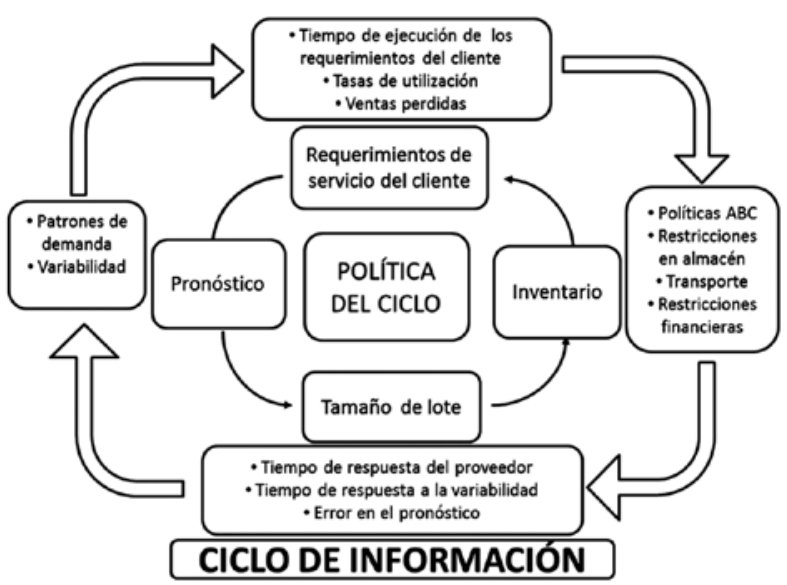

Ilustración 1. Ciclo Ventas - Producción- Compras²

En la Ilustración 1 se aprecia cómo a partir de patrones de demanda y variabilidad de la misma, las empresas establecen tiempos de entrega a clientes y tasas de respuesta requeridas, pero también el riesgo de ventas pérdidas. Esta información considera además, restricciones de almacenamiento y transporte, financieras, combinación de referencias de inventario en términos de alta, media y baja rotación (clasificación $\mathrm{ABC}$ ) y obsolescencia.

En cuanto a políticas, los requerimientos de los clientes son la base del presupuesto de ventas, sobre el que se determinarán tamaños de lotes de producción o lotes de compras, lo que incide a su vez en la determinación de la composición y nivel del inventario, para al final cumplir con las órdenes de clientes, es decir satisfacer la demanda. De esta forma, se sincronizan los volúmenes o tasas de oferta y demanda, para nivelar y regularizar el flujo de inventarios en la empresa, evitando desviaciones de éste por exceso o por defecto. Su exceso producirá sobrecostos de almacenamiento, costo financiero por el dinero inmovilizado y riesgo de obsolescencia. Su escasez, ventas pérdidas y baja credibilidad ante los clientes.

En consecuencia, el punto de partida del ciclo debe ser la composición y volumen de demanda, de lo cual se obtiene información básica para decisiones de producción y compras, respecto al tamaño de lotes a producir o adquirir y niveles de inventarios a mantener, que a su vez son afectados por el nivel de servicio al cliente o tasa de cumplimiento de órdenes de cliente (Lawrence, 1999).

El ciclo se repite para cada producto de la composición de demanda y cada periodo de tiempo, lo que requiere un monitoreo permanente para identificar variaciones y ajustar la composición y volumen de oferta, buscando generar una respuesta efectiva y oportuna ante los requerimientos de los clientes, traducida en mejores utilidades para la empresa. Los inventarios pueden aumentar para unas referencias y escasear para otras, de acuerdo con las contingencias y variaciones de la demanda y el abastecimiento, por lo que es indispensable contar con información permanente para poder reaccionar oportunamente (Lawrence, 1999). Las políticas administrativas y la disponibilidad de información para la toma de decisiones son las claves del proceso. En resumen, es necesario identificar un patrón de demanda para cada producto o familia de productos, que permita ajustar la oferta al mismo.

La compañía podrá incidir en el comportamiento de aquellos factores que estén bajo su control como el costo de los productos o el costo del manejo de inventarios, pero deberá adaptarse y responder ante aquellos que no controla, como es el caso de los tiempos de reposición de los proveedores o la variabilidad de la demanda. Respecto a esta última, la teoría recomienda el uso de modelos matemáticos combinado con el conocimiento del mercado y la experiencia de la dirección, lo que ha demostrado ser más efectivo que el uso de solo el modelo matemático o solo la experiencia e intuición (Lawrence, 1999).

2 Fuente: Lawrence (1999, 43-46). 
En cuanto los proveedores, dado que cada uno tiene sus propios tiempos, condiciones y características de respuesta, se requiere un estudio detallado de los mismos (Lawrence, 1999) para diseñar acciones conducentes a administrar adecuadamente la gestión de compras, elaborando planes y programas que respondan a la demanda de clientes, consideren los tiempos de reabastecimiento brindados por los proveedores $y$ permitan nivelar los inventarios, para evitar excesos o defectos, que en cualquier caso, tienen un impacto sobre la sostenibilidad económica de la empresa.

Ante los cambios de la composición y volumen de la demanda de clientes que se puedan derivar de fenómenos como la pobreza oculta, es indiscutible que las empresas deben fortalecer su capacidad de gestión para sincronizar sus volúmenes o tasas y estructura de oferta con los volúmenes o tasas y estructura de la demanda, y tener la flexibilidad necesaria en sus operaciones para adaptar su estructura de oferta a los cambios intempestivos de los requerimientos de la demanda.

\section{Antecedentes del estudio de la pobreza en Colombia}

En Colombia existe una estratificación socioeconómica consistente en una clasificación de los domicilios o viviendas a partir de sus características físicas y el acceso a infraestructura de servicios públicos, vías, salud, educación y recreación, dividida en seis grupos o estratos (Departamento Nacional de Planeación, 2010), donde cada vivienda se clasifica dentro de uno de estos, lo que sirve de base para el cobro de tarifas de servicios públicos domiciliarios, $e$ impuestos asociados al inmueble, siendo las tarifas más bajas las correspondientes a estratos 1 y 2 y las más altas, a estratos 5 y 6 .

Se establece entonces una relación entre la población de escasos recursos o clase baja con los estratos uno y dos, clase media con estratos tres y cuatro y clase media alta y alta con estratos cinco y seis, asociando cada estrato con la capacidad económica de su población (Uribe, Vasquez \& Pardo, 2006, 69-94).
Sin embargo, según Sandra Jaramillo, ex Alcaldesa de la Localidad de Teusaquillo en Bogotá, la pobreza no necesariamente coincide con el estrato. El que una persona viva en estrato 1 o 2 no significa que se halle en situación de pobreza, y la persona que vive en estrato 4 no necesariamente tiene capacidad y holgura económica ${ }^{3}$.

Por otra parte, el Departamento Nacional de Estadística DANE, utiliza la encuesta de calidad de vida ECV para "cuantificar y caracterizar las condiciones de vida de los pobres y de los no pobres" (DANE, 2010a). La última encuesta fue aplicada en 2008 en todo el país, haciendo uso del muestreo probabilístico, estratificado, de conglomerados y polietápico, a 13.600 hogares en todo el país (DANE, 2010b).

Otro instrumento es el índice de necesidades básicas insatisfechas NBI que "busca determinar si las necesidades básicas de la población se encuentran cubiertas, y tiene relación con indicadores de vivienda". Los grupos de población que no alcanzan un umbral mínimo o puntaje establecido, son clasificados como pobres (DANE, 2009).

Complementariamente existe, el sistema de identificación y clasificación de potenciales beneficiarios para programas sociales SISBEN, el cual es un sistema de información diseñado por el Estado colombiano para "identificar a las personas y familias más necesitadas y vulnerables y ordenarlas de acuerdo con su condición socio económica, como potenciales beneficiarios para programas sociales" (Secretaria de Planeación, 2009)

Estos instrumentos comparten el hecho de que ninguno profundiza en el tratamiento individual de personas y hogares, ni en aspectos de lo privado que tienen relación con la pobreza, que es el espacio donde se da el fenómeno de pobreza oculta.

Al respecto, en el año 2008 se publicaron los resultados de la investigación patrocinada por la Alcaldía Local de Teusaquillo, titulada "Indicadores de pobreza oculta de la Localidad de Teusaquillo"

3 (www.samuelalcalde.com). 
elaborada por Hernán Pabón en el año 2007, los cuales incluyeron un diagnóstico sobre la pobreza en la Localidad. Como parte de los hallazgos, el estudio encontró discrepancias entre las cifras de pobreza reportadas a través de la encuesta de calidad de vida ECV, el índice de necesidades básicas insatisfechas NBI y la encuesta del SISBEN, por lo que una de sus más importantes recomendaciones para futuras investigaciones fue el realizar estudios específicos para focalizar problemas y variables a nivel de barrios y manzanas, dado que cada sitio tiene condiciones socioeconómicas y urbanas que los caracterizan, con énfasis en la revisión de manera individualizada de hogares y personas (Pabón, 2008).

Haciendo eco de estas recomendaciones, esta investigación aplicó una metodología que permitió ese acercamiento individual a jefes de hogar y empresarios, puesto que las condiciones propias del fenómeno de pobreza oculta al interior de cada hogar y como esta situación afecta los hábitos de compra y consumo de bienes y servicios, solo son detectables a través de la aplicación de métodos de investigación, que permitan profundizar en el día a día de los hogares.

\section{Caracterización de la población de Usaquén}

De acuerdo con estudio efectuado por la Cámara de Comercio de Bogotá (2006), en Usaquén predominan las clases socioeconómicas media y alta: el $32,3 \%$ de predios son estrato 4 y ocupan la mayor parte del área urbana local, el 24,8\% pertenece a predios de estrato 6 , el estrato 3 representa el $21,1 \%$, el 14,9\% corresponde a predios de estrato 5 . En razón de esta distribución de área por estratos, la probabilidad de que exista el fenómeno de pobreza oculta en la Localidad, en clases medias y altas, es altamente posible.

Usaquén está distribuido en nueve UPZ y 134 barrios (Listado de barrios por UPZ, 2009) y contaba para el 2006 con una población de 441 mil personas donde el promedio de personas por hogar era de 3.2 (Cámara de Comercio de Bogotá, 2006). La población mayor de cinco años tiene en promedio
10.9 años de educación, y en todos los grupos de edad, la localidad cuenta con niveles educativos significativamente superiores a los de toda la ciudad de Bogotá.

La población económicamente activa, PEA, que reside en Usaquén, tiene en su mayoría educación superior $(63,6 \%)$. La tasa de ocupación de la localidad $(57,6 \%)$ supera la de la ciudad $(55,1 \%)$, y es la sexta más alta entre las localidades. En cuanto a la posición ocupacional de los empleados que viven en la localidad, el 46,8\% son empleados de empresa particular, el 16,9\% trabajadores por cuenta propia y el $11 \%$ profesional independiente.

\section{Tipos de empresas y tejido empresarial de Usaquén}

Según el artículo segundo de la Ley 590 del 10 de julio de 2000 la siguiente es la definición de micro, pequeña y mediana empresa:

"Para todos los efectos, se entiende por micro, pequeña y mediana empresa, toda unidad de explotación económica, realizada por persona natural o jurídica, en actividades empresariales, agropecuarias, industriales, comerciales o de servicios, rural o urbana" (Congreso de la República de Colombia, 2000).

- Microempresa: unidades económicas con no más de 10 trabajadores, cuyos activos totales son inferiores a quinientos uno (501) salarios mínimos mensuales legales vigentes.

- Pequeña empresa: unidades económicas que poseen entre once (11) y cincuenta (50) trabajadores, cuyos activos totales deben estar entre quinientos uno (501) y menos de cinco mil uno (5.001) salarios mínimos mensuales legales vigentes.

- Mediana empresa: unidades económicas que poseen entre cincuenta y uno (51) y doscientos (200) trabajadores, cuyos activos totales se encuentran entre cinco mil uno (5.001) y quince mil (15.000) salarios mínimos mensuales legales vigentes. 
Zerda \& Rincón (1998, 21-41), en su análisis sobre la transformación conceptual de la pequeña y mediana industria en el mundo occidental, clasifican estas en dos categorías: subsistencia y dinamismo). La empresa de subsistencia tiene procesos tecnológicos simples, fuerza de trabajo no calificada, baja capacidad empresarial, no cuentan con una estructura organizacional que motive la innovación, producen bienes de consumo y no tienen incidencia en el mercado y se encuentran en punto de equilibrio económico lo que no les permite ahorrar o reinvertir, por lo que se estancan La empresas dinámicas por otra parte, utilizan tecnologías (blandas y duras) flexibles, fuerza de trabajo calificada, tienen alta capacidad gerencial, son innovadoras en procesos y/o productos, pueden llegar a producir bienes de inversión y tener incidencia en el mercado y tienen altas tasas de ahorro y reinversión, siendo su principal fuente de financiación son sus propios fondos.

Las características comunes entre los dos tipos, son: poco capital que se mantiene en un nivel constante, uso intensivo de la fuerza de trabajo y gran flexibilidad en los costos que permite a este tipo de empresas, ajustarse a los ciclos económicos de prosperidad y recesión sin llegar a la sobreproducción.

Sin embargo, la pyme de subsistencia "se apoya en un conjunto de aspectos que impiden pensarla como una unidad capaz de resistir la competencia o de generar efectos económicos" (Zerda \& Rincón, 1998, 37) y su cualidad es la de generar empleo en el corto plazo. Por esto mismo, este tipo de empresas están más expuestas a perder su sostenibilidad económica, lo que deriva, adicionalmente, en la pérdida de los empleos que generan, cuando se presentan variaciones que disminuyen la demanda de algunos bienes y servicios.

Dado que en el año 2004 se encontró que en la Localidad de Usaquén, según el tamaño de las empresas, había 15.194 microempresas, que representaban el $80 \%$ de las empresas establecidas en la localidad y las PYMES el 19\% (Cámara de Comercio de Bogotá, 2006), esta distribución hace que el tejido empresarial de la localidad sea sensible a los cambios de la demanda, en lo que a sostenibilidad económica se refiere.

\section{Metodología}

La investigación es cualitativa, exploratoria y descriptiva, por cuanto se buscó establecer si hay o no pobreza oculta en los hogares de clases medias y altas en la Localidad de Usaquén y de existir, cuáles son sus características y cómo este fenómeno puede estar afectando la sostenibilidad económica de las pequeñas y medianas empresas de la misma localidad.

Por esto se estudiaron dos poblaciones: hogares estratos 3, 4 y 5 de la localidad de Usaquén y micros, pequeñas y medianas empresas de la localidad, específicamente de los sectores de Lijacá, San Cristobal Norte, Usaquén, Cedritos, Santa Barbará, Santa Beatriz y Danubio, con más de un año de funcionamiento.

Para mejorar la calidad del estudio cualitativo, se utilizó la triangulación (Okuda \& Gómez, 2005, 118-124), método consistente en la aplicación de métodos cualitativos, de los cuales se aplicaron entrevistas semi- estructuradas a empresarios y grupos focales a jefes de hogar estrato 4 , y de cuyos resultados se extrajeron las categorías de respuesta para la construcción de las encuestas de preguntas de formato cerrado y métodos cuantitativos, utilizando el muestreo por conglomerados para la aplicación de las encuestas a las muestras de las dos poblaciones de estudio. Esta metodología permite observar la totalidad del problema desde diferentes ángulos y perspectivas minimizando el sesgo y aumentando la validez y consistencia de los hallazgos.

El tamaño de muestra de hogares fue de 129 unidades, con un intervalo de confianza del $95 \%$ y porcentaje de error del $8.6 \%$. El tamaño de muestra de empresas fue de 86 unidades con un intervalo de confianza del $95 \%$ y un porcentaje de error del $10.6 \%$. Para la selección de las unidades de muestreo, se dependió de la voluntad de las personas contactadas de colaborar con el estudio.

Para el estudio de hogares, se seleccionó la variable nominal proporcional referida a si el hogar enfrenta o no pobreza oculta. Las variables descriptivas de la encuesta fueron: cambio cantidad de compra, cambio sitio de compra, lugar de compra, razones de cambio sitio de compra, categorías de bienes/ 
servicios con dificultades de pago, y causas de la disminución del ingreso familiar.

Para el estudio de empresas, se seleccionó la variable de estudio nominal proporcional referida a si la pobreza impacta o no la sostenibilidad económica de la empresa. Las variables descriptivas de la encuesta fueron: Productos/servicios con aumento en ventas, causas del aumento en ventas, productos/servicios con disminución en ventas, causas de la disminución en ventas, tipo de clientes atendidos, estrategias para mantener clientes, comportamiento de las ventas, disponibilidad de inventarios, disponibilidad de capacidad, comportamiento de la utilidad, comportamiento de la cantidad de clientes, cambio cantidad de compra clientes, cambio frecuencia de compra clientes, cambio productos comprados por clientes, ajustes a la oferta solicitados por los clientes, razones de pérdida de clientes, afectación por la recesión y modo de afectación de la recesión.

Para el estudio de hogares se llevaron a cabo dos grupos focales, uno de damas y otro de caballeros de estrato 4, cada uno con duración de hora y medio en promedio. Y se aplicó la encuesta a jefes de hogar habitantes de estratos 3, 4 y 5 de la localidad de Usaquén.
Para el estudio de empresas, se aplicaron entrevistas semi estructuradas a empresarios de la localidad, y posteriormente la encuesta a empresarios, de acuerdo con una base de datos de empresas por sectores, que combinó información de la base de datos de la Unidad Local de Desarrollo Empresarial ULDE de la Localidad de Usaquén e información sobre direcciones de sectores comerciales $e$ industriales con concentración de pequeñas y medianas empresas.

Tanto la encuesta de hogares, como la de empresas fueron procesadas en SPSS $®$ Versión 14, para obtener la distribución de frecuencias de las categorías de respuesta, único análisis aplicable a variables nominales.

\section{Resultados}

8.1 Grupos focales jefes de hogar y entrevistas empresarios. Variables que caracterizan la pobreza oculta en los hogares de estratos 3,4 y 5

En los grupos focales, los jefes de hogar manifestaron haber reducido el uso del carro particular, sa-

Tabla 2. Variables nominales como resultado de la investigación cualitativa ${ }^{4}$

\begin{tabular}{|l|l|l|}
\hline \multicolumn{1}{|c|}{ Variables nominales } & \multicolumn{1}{|c|}{ Descripción } & \multicolumn{1}{|c|}{ Hallazgos } \\
\hline Cambios de hábitos de consumo & $\begin{array}{l}\text { Permite categorizar los cambios en la } \\
\text { mezcla de bienes y servicios consumi- } \\
\text { dos, lo que muestra los cambios en la } \\
\text { estructura de demanda. }\end{array}$ & $\begin{array}{l}\text { Los cambios más determinantes son } \\
\text { la eliminación del uso de productos } \\
\text { considerados como suntuarios, inclu- } \\
\text { yendo disminución del uso del vehículo } \\
\text { particular. }\end{array}$ \\
\hline Cambios de hábitos de compra & $\begin{array}{l}\text { Permite categorizar los cambios de lu- } \\
\text { gar, frecuencia, cantidad y mezcla de } \\
\text { compra, lo que incide en cambios de } \\
\text { estructura de demanda. }\end{array}$ & $\begin{array}{l}\text { Los cambios más determinantes son el } \\
\text { buscar un sitio donde se obtenga ma- } \\
\text { yor economía en las compras, así sea } \\
\text { de otro sector o estrato. }\end{array}$ \\
\hline Dificultades económicas & $\begin{array}{l}\text { Permite categorizar los eventos que } \\
\text { disminuyen los ingresos del núcleo } \\
\text { familiar y afectan su capacidad de } \\
\text { compra y pago de diversos bienes y } \\
\text { servicios }\end{array}$ & $\begin{array}{l}\text { El más representativo es la pérdida del } \\
\text { empleo de alguno de los miembros de } \\
\text { la familia básica o ampliada. }\end{array}$ \\
\hline
\end{tabular}

4 Fuente: Elaboración propia a partir de resultados de la investigación. 
lón de belleza, vacaciones, empleada doméstica, alimentos, productos de lujo o cambio de los hijos de colegio privado a público. Cambiaron de sitio de compra buscando precios bajos, cercanía y presentaciones diversos tamaños. Enfrentan dificultades económicas por la pérdida del empleo de alguno de los miembros de la familia, la reducción de las ventas de la empresa o negocio propio, o el retorno de hijos adultos al hogar paterno por falta de empleo. Algunos han vendido su vivienda en estrato 5 para pasar al 3, o han prescindido del vehículo particular.

De estos resultados, se obtuvieron las variables nominales que caracterizan la pobreza oculta en estos hogares, las cuales se observan en la tabla 2 . Al cruzar las respuestas se encontró que hay coincidencia entre las respuestas de los jefes de hogar y los empresarios entrevistados. Mientras los jefes de hogar manifiestan que han disminuido el uso del vehículo particular, los empresarios expresan que los clientes están ahorrando en mantenimiento de sus vehículos. Tanto los jefes de hogar como los empresarios de salas de belleza coinciden en la disminución de la frecuencia del uso de este servicio por parte de los clientes. Además los jefes de hogar comentan que cambian de sitio de compra en busca de menores precios y presentaciones personales, los empresarios dicen que los clientes piden descuentos y precios más bajos. Finalmente, los jefes de hogar manifiestan que pasan de comprar en negocios de su estrato, a negocios de menor estrato y los empresarios de estrato 3 e incluso 2 , confirman que han llegado nuevos clientes procedentes de estratos 4 y 5 .

\subsection{Encuesta hogares. Cambios hábitos de compra y composición demanda hogares estratos 3,4 y 5}

Con base en los resultados de los grupos focales, y las preguntas relacionadas ya establecidas en la encuesta de calidad de vida ECV y el índice de necesidades básicas insatisfechas NBI del DANE, se diseñaron las preguntas de la encuesta de hogares, considerando la canasta familiar por este último. Una vez aplicada la encuesta a los jefes de hogar de la muestra, pre- sentaron la mayor disminución de compras, en las siguientes categorías de productos: Ropa $65,9 \%$, calzado $64,3 \%$, enlatados $44,2 \%$, confitería $43,4 \%$, salsamentaría $38 \%$, bebidas alcohólicas $34,1 \%$, frutas $32,6 \%$, verduras $29,5 \%$, carnes $34,9 \%$, pan $38,8 \%$, granos $31 \%$ y pastas $31,8 \%$. Los hogares encuestados a su vez, dejaron de comprar en las siguientes proporciones: Cigarrillos $62,8 \%$, bebidas alcohólicas $41,9 \%$ y confitería $30,2 \%$ (tabla 3 ).

Tabla 3. Disminución en compra de ropa ${ }^{5}$

\begin{tabular}{|l|c|c|c|c|}
\hline & Frequency & Percent & $\begin{array}{c}\text { Valid } \\
\text { Percent }\end{array}$ & $\begin{array}{c}\text { Cumulative } \\
\text { Percent }\end{array}$ \\
\hline misma cantidad & 29 & 22,5 & 22,5 & 22,5 \\
\hline bajó cantidad & 85 & 65,9 & 65,9 & 88,4 \\
\hline aumentó cantidad & 4 & 3,1 & 3,1 & 91,5 \\
\hline dejo de comprar & 11 & 8,5 & 8,5 & 100 \\
\hline Total & 129 & 100 & 100 & \\
\hline
\end{tabular}

Las categorías de productos que presentan los mayores porcentajes de cambio de sitio de compra, de acuerdo con el número de respuestas recibidas para comprar en el mismo sitio o cambiar el sitio de compras, son: frutas $44,1 \%$, verduras $43,1 \%$ cárnicos $45,5 \%$, ropa $51,6 \%$ y calzado $45,9 \%$. Esto confirma la información recibida en los grupos focales. En general, hubo un promedio de cambio de sitio de compra en el 33,2\% de los casos encuestados.

Tabla 4. Cambio de sitio de compra de las frutas ${ }^{6}$

\begin{tabular}{|l|c|c|c|c|}
\hline & Frequency & Percent & $\begin{array}{c}\text { Valid } \\
\text { Percent }\end{array}$ & $\begin{array}{c}\text { Cumulative } \\
\text { Percent }\end{array}$ \\
\hline Cambio sitio de compra & 30 & 23,3 & 23,3 & 23,3 \\
\hline $\begin{array}{l}\text { Compra en el mismo } \\
\text { sitio }\end{array}$ & 38 & 29,5 & 29,5 & 52,7 \\
\hline $\begin{array}{l}\text { Compra en hipermerca- } \\
\text { dos y/0 almacenes de } \\
\text { cadena }\end{array}$ & 26 & 20,2 & 20,2 & 72,9 \\
\hline $\begin{array}{l}\text { Compra en supermerca- } \\
\text { dos de barrio }\end{array}$ & 15 & 11,6 & 11,6 & 84,5 \\
\hline
\end{tabular}

5 Fuente: Elaboración propia a partir de resultados de la investigación. Salida SPSS®14.
6 Fuente: Elaboración propia a partir de resultados de la investigación. Salida SPSS 14. 


\begin{tabular}{|l|c|c|c|c|}
\hline $\begin{array}{l}\text { Compra en plaza de mer- } \\
\text { cado o mercado movil }\end{array}$ & 5 & 3,9 & 3,9 & 88,4 \\
\hline $\begin{array}{l}\text { Compra en tienda de } \\
\text { barrio }\end{array}$ & 6 & 4,7 & 4,7 & 93 \\
\hline Compra en codabas & 7 & 5,4 & 5,4 & 98,4 \\
\hline Compra en corabastos & 2 & 1,6 & 1,6 & 100 \\
\hline Total & 129 & 100 & 100 & \\
\hline
\end{tabular}

La razón de cambio de sitio de compra más representativa fue búsqueda de precios bajos con $49,6 \%$ de las respuestas, corroborando lo dicho en los grupos focales.

Tabla 5. Razones de cambio de sitio de compra ${ }^{7}$

\begin{tabular}{|l|c|c|c|}
\hline \multicolumn{1}{|c|}{ Razones de cambio } & Frecuencia & $\%$ & $\%$ acumulado \\
\hline Cercanía sitio de compra actual & 24 & 18,6 & 24,8 \\
\hline Precios más bajos en sitio actual & 64 & 49,6 & 74,4 \\
\hline Surtido del sitio actual & 9 & 7 & 81,4 \\
\hline Variedad de tamaños productos & 17 & 13,2 & 94,6 \\
\hline Diferentes alternativas de pago & 7 & 5,4 & 100 \\
\hline Total & 129 & 100 & \\
\hline
\end{tabular}

Respecto a los ingresos familiares, estos disminuyeron por las siguientes razones: pérdida del empleo de uno de los integrantes $(28,7 \%)$, disminución de ventas de la empresa familiar (16\%) y cambio a un empleo con menor remuneración (13\%). Se puede apreciar que el mayor porcentaje de afectación del ingreso familiar se deriva de cambios en el empleo, sea por pérdida o deterioro del mismo.

Tabla 6. Eventos que disminuyeron los ingresos familiares en los dos últimos años ${ }^{8}$

\begin{tabular}{|l|c|c|c|}
\hline \multicolumn{1}{|c|}{ Eventos } & Frecuencia & $\%$ & \% acumulado \\
\hline $\begin{array}{l}\text { Pérdida del empleo del jefe de } \\
\text { hogar }\end{array}$ & 19 & 14,7 & 17,1 \\
\hline $\begin{array}{l}\text { Pérdida de empleo de uno de los } \\
\text { miembros de la familia }\end{array}$ & 18 & 14 & 31 \\
\hline $\begin{array}{l}\text { Disminución en las ventas de la } \\
\text { empresa familiar }\end{array}$ & 21 & 16,3 & 47,3 \\
\hline Perdidas de la empresa familiar & 8 & 6,2 & 53,5 \\
\hline
\end{tabular}

\begin{tabular}{|l|c|c|c|}
\hline Enfermedad del jefe de hogar & 6 & 4,7 & 58,1 \\
\hline $\begin{array}{l}\text { Enfermedad de uno de los miem- } \\
\text { bros de la familia }\end{array}$ & 13 & 10,1 & 68,2 \\
\hline Fallecimiento del jefe de hogar & 5 & 3,9 & 72,1 \\
\hline Separación & 8 & 6,2 & 78,3 \\
\hline Divorcio & 3 & 2,3 & 80,6 \\
\hline $\begin{array}{l}\text { Cambio de empleo a uno de menor } \\
\text { salario }\end{array}$ & 17 & 13,2 & 93,8 \\
\hline $\begin{array}{l}\text { Hacerse cargo de los padres por } \\
\text { dificultades económicas de ellos }\end{array}$ & 4 & 3,1 & 96,9 \\
\hline $\begin{array}{l}\text { Hacerse cargo de parientes por } \\
\text { dificultades económicas de estos }\end{array}$ & 4 & 3,1 & 100 \\
\hline Total & 129 & 100 & \\
\hline
\end{tabular}

Por otra parte, los hogares que vieron afectados sus ingresos por la pérdida del empleo de uno de los miembros de la familia, el $63 \%$ cambió de sitio de compra por la búsqueda de precios más bajos. De los hogares afectados por disminución en las ventas de la empresa familiar, el 52,4\% cambió de sitio de compra por la misma razón. El estrato más afectado por el cambio a un empleo de menor salario fue el cuatro (4), con un $16,7 \%$, seguido por la pérdida del empleo del jefe de hogar con un $15 \%$.

\subsection{Encuesta a empresas. Variaciones de la demanda y ajustes de la oferta}

Los empresarios por su parte respondieron que se han presentado aumentos en las ventas de algunos de los bienes y servicios ofertados, entre los cuales emergen categorías no consideradas en la canasta, como son: alitas de pollo, almuerzo corriente, arreglo ropa, cafés, cerveza, chance, dulces, esferos, fotocopias, impermeables, materiales de construcción, planificación familiar, recargas de celulares, reparaciones, repostería, ropa bebé, salsas, tamales, teléfonos celulares, tintes y tinturas.

En los casos en que las ventas aumentaron, esto se debió en un $43 \%$ a nuevos clientes en tanto que el $15,1 \%$ lo hizo por el aumento en la cantidad comprada por los clientes habituales (tabla 7).

\footnotetext{
Fuente: Elaboración propia a partir de resultados de la investigación. Adaptada de salida SPSS®14.

Fuente: Elaboración propia a partir de resultados de la investigación. Adaptada de salida SPSS®14.
} 
Tabla 7. Razones del aumento en ventas ${ }^{9}$

\begin{tabular}{|l|c|c|c|}
\hline \multicolumn{1}{|c|}{$\begin{array}{c}\text { Razones aumento en } \\
\text { ventas }\end{array}$} & Frecuencia & $\%$ & $\%$ acumulado \\
\hline Nuevos Clientes & 37 & 43 & 43 \\
\hline $\begin{array}{l}\text { Aumento cantidad comprada por } \\
\text { clientes habituales }\end{array}$ & 13 & 15,1 & 58,1 \\
\hline Apertura Nuevo Local & 6 & 7 & 65,1 \\
\hline Oferta nuevos productos & 10 & 11,6 & 76,7 \\
\hline Otros & 7 & 8,1 & 84,9 \\
\hline NA & 13 & 15 & 100 \\
\hline Total & 86 & 100 & \\
\hline
\end{tabular}

Los productos y servicios con disminución en ventas son: almuerzo ejecutivo, baloto, bebidas frías (granizados), bloqueadores, bolsos, bota en cuero de seguridad, botones, buzos, $\mathrm{CD}$, cepillado, champú, Chocolates, chorizos, cintas, comidas a la carta, DVD, enlatados, gaseosas, granos, hilo, lanas, leche en polvo, licores, medicinas, multi-vitamínicos, pañales, papelería, pechugas, pedicure, productos de aseo, productos de belleza, queso, regalos, ropa, telefonía, tintes, yogurt.

El 25,6\% de los empresarios reporta disminución en ventas por baja en los pedidos de los clientes habituales y el $34,9 \%$ varias razones, entre las cuales se encuentran, aspectos relacionados con el clima, la economía, el estado de las vías y la capacidad de compra de los clientes, tal como se indica en seguida: Estado del tiempo, pico y placa, apertura de competencia, economía, rotación por temporadas, precio, competencia desleal, arreglo de calles, cierre de una vía, cierre de zonas de parqueo, falta de dinero del cliente (tabla 8).
Tabla 8. Causas de la disminución de las ventas ${ }^{10}$

\begin{tabular}{|l|c|c|c|}
\hline \multicolumn{1}{|c|}{ Causas } & Frecuencia & $\%$ & $\%$ acumulado \\
\hline Pérdida de Clientes & 11 & 12,8 & 12,8 \\
\hline $\begin{array}{l}\text { Disminución pedidos Clientes } \\
\text { habituales }\end{array}$ & 22 & 25,6 & 38,4 \\
\hline $\begin{array}{l}\text { Reducción de productos 0 } \\
\text { servicios ofrecidos }\end{array}$ & 4 & 4,7 & 43 \\
\hline Otros & 30 & 34,9 & 77,9 \\
\hline NA & 19 & 22,1 & 100 \\
\hline Total & 86 & 100 & \\
\hline
\end{tabular}

Como estrategias para conservar los clientes el $24,4 \%$ de los empresarios encuestados reporta aumento del surtido de productos, en tanto que el $22.1 \%$ de los mismos ha hecho mejoras a sus instalaciones (Ver tabla 9).

Tabla 9. Estrategias para mantener clientes ${ }^{11}$

\begin{tabular}{|l|c|c|c|}
\hline \multicolumn{1}{|c|}{ Estrategias } & Frecuencia & $\%$ & $\%$ acumulado \\
\hline $\begin{array}{l}\text { Congela los precios } \\
\text { de los productos }\end{array}$ & 13 & 15,1 & 15,1 \\
\hline $\begin{array}{l}\text { Mejora instalaciones } \\
\text { del servicio }\end{array}$ & 19 & 22,1 & 37,2 \\
\hline $\begin{array}{l}\text { Aumenta surtido } \\
\text { de productos }\end{array}$ & 21 & 24,4 & 61,6 \\
\hline $\begin{array}{l}\text { Mejora exhibición } \\
\text { de productos }\end{array}$ & 18 & 20,9 & 82,6 \\
\hline $\begin{array}{l}\text { Surte ante agotados } \\
\text { sin importar el costo }\end{array}$ & 10 & 11,6 & 94,2 \\
\hline Otro & 5 & 5,8 & 100 \\
\hline Total & 86 & 100 & \\
\hline
\end{tabular}

Respecto al tipo de clientes atendidos, el $87,2 \%$ de los empresarios encuestados atienden clientes del estrato habitual mientras que un $10,5 \%$ atiende clientes de un estrato más alto (tabla 10).

\footnotetext{
9 Fuente: Elaboración propia a partir de resultados de la investigación. Adaptada de salida SPSS 14 .

10 Fuente: Elaboración propia a partir de resultados de la investigación. Adaptada de salida SPSS®14.

11 Fuente: Elaboración propia a partir de resultados de la investigación. Adaptada de salida SPSS®14.
} 
Tabla 10. Tipo de clientes atendidos ${ }^{12}$

\begin{tabular}{|l|c|c|c|c|}
\hline & Frequency & Percent & $\begin{array}{c}\text { Valid } \\
\text { Percent }\end{array}$ & $\begin{array}{c}\text { Cumulative } \\
\text { Percent }\end{array}$ \\
\hline $\begin{array}{l}\text { Estrato } \\
\text { habitual }\end{array}$ & 75 & 87,2 & 87,2 & 87,2 \\
\hline $\begin{array}{l}\text { Estrato más } \\
\text { Alto }\end{array}$ & 9 & 10,5 & 10,5 & 97,7 \\
\hline $\begin{array}{l}\text { Estrato más } \\
\text { bajo }\end{array}$ & 2 & 2,3 & 2,3 & 100 \\
\hline Total & 86 & 100 & 100 & \\
\hline
\end{tabular}

Por otra parte, el 39,5\% de los empresarios encuestados reporta aumento en ventas en tanto que el $32,6 \%$ enfrentan una disminución. Se observa en la tabla 11 una distribución uniforme entre el aumento, el mantenimiento y la disminución de las ventas.

Tabla 11. Tendencias del comportamiento de las ventas ${ }^{13}$

\begin{tabular}{|l|c|c|c|}
\hline \multicolumn{1}{|c|}{ Tendencia } & Frecuencia & $\%$ & \% acumulado \\
\hline $\begin{array}{l}\text { Definitivamente au- } \\
\text { mentan }\end{array}$ & 3 & 3,5 & 3,5 \\
\hline Aumentan & 11 & 12,8 & 16,3 \\
\hline Se mantienen & 34 & 39,5 & 55,8 \\
\hline Disminuyen & 28 & 32,6 & 88,4 \\
\hline $\begin{array}{l}\text { Definitivamente dis- } \\
\text { minuyen }\end{array}$ & 10 & 11,6 & 100 \\
\hline Total & 86 & 100 & \\
\hline
\end{tabular}

En cuanto los inventarios (ver tabla 12 ), el $58,1 \%$ de los empresarios mantienen sus niveles de inventario, en tanto que el $18,6 \%$ presentan una disminución. Esto indica una tendencia a mantener disponibilidad de productos para atender la demanda.
Tabla 12. Disponibilidad de inventarios ${ }^{14}$

\begin{tabular}{|l|c|c|c|c|}
\hline & Frequency & Percent & $\begin{array}{c}\text { Valid } \\
\text { Percent }\end{array}$ & $\begin{array}{c}\text { Cumulative } \\
\text { Percent }\end{array}$ \\
\hline $\begin{array}{l}\text { Definitivamente } \\
\text { aumento }\end{array}$ & 7 & 8,1 & 8,1 & 8,1 \\
\hline Aumento & 6 & 7 & 7 & 15,1 \\
\hline Se mantiene & 50 & 58,1 & 58,1 & 73,3 \\
\hline Disminuyo & 16 & 18,6 & 18,6 & 91,9 \\
\hline $\begin{array}{l}\text { Definitivamente } \\
\text { disminuyo }\end{array}$ & 7 & 8,1 & 8,1 & 100 \\
\hline Total & 86 & 100 & 100 & \\
\hline
\end{tabular}

En cuanto la capacidad instalada (ver tabla 13), el $52,3 \%$ de los empresarios mantiene su capacidad en tanto que el $19,8 \%$ de los mismos disminuye su capacidad y el $12,8 \%$ la aumenta. Estas variaciones dependerán de los cambios en las ventas que cada compañía enfrente.

Tabla 13. Disponibilidad de capacidad ${ }^{15}$

\begin{tabular}{|l|c|c|c|c|}
\hline & Frequency & Percent & $\begin{array}{c}\text { Valid } \\
\text { Percent }\end{array}$ & $\begin{array}{c}\text { Cumulative } \\
\text { Percent }\end{array}$ \\
\hline $\begin{array}{l}\text { Definitivamente } \\
\text { aumento }\end{array}$ & 3 & 3,5 & 3,5 & 3,5 \\
\hline Aumento & 11 & 12,8 & 12,8 & 16,3 \\
\hline Se mantiene & 45 & 52,3 & 52,3 & 68,6 \\
\hline Disminuy0 & 17 & 19,8 & 19,8 & 88,4 \\
\hline $\begin{array}{l}\text { Definitivamente } \\
\text { disminuy0 }\end{array}$ & 10 & 11,6 & 11,6 & 100 \\
\hline Total & 86 & 100 & 100 & \\
\hline
\end{tabular}

Por otra parte, en la tabla 14 se ve que para el $36 \%$ de las empresas encuestadas se mantienen las utilidades mientras que para el $33,7 \%$ disminuyen, lo cual se relaciona con el aumento o disminución de las ventas.

12 Fuente: Elaboración propia a partir de resultados de la investigación. Salida SPSS®14.

13 Fuente: Elaboración propia a partir de resultados de la investigación. Adaptada de salida SPSS®14.

14 Fuente: Elaboración propia a partir de resultados de la investigación. Salida SPSS®14.

15 Fuente: Elaboración propia a partir de resultados de la investigación. Salida SPSS®14. 
Tabla 14. Comportamiento de la utilidad ${ }^{16}$

\begin{tabular}{|l|c|c|c|c|}
\hline & Frequency & Percent & $\begin{array}{c}\text { Valid } \\
\text { Percent }\end{array}$ & $\begin{array}{c}\text { Cumulative } \\
\text { Percent }\end{array}$ \\
\hline $\begin{array}{l}\text { Definitivamente } \\
\text { aumento }\end{array}$ & 5 & 5,8 & 5,8 & 5,8 \\
\hline Aumento & 10 & 11,6 & 11,6 & 17,4 \\
\hline Se mantiene & 31 & 36 & 36 & 53,5 \\
\hline Disminuyo & 29 & 33,7 & 33,7 & 87,2 \\
\hline $\begin{array}{l}\text { Definitivamente } \\
\text { disminuyo }\end{array}$ & 11 & 12,8 & 12,8 & 100 \\
\hline Total & 86 & 100 & 100 & \\
\hline
\end{tabular}

En cuanto a los clientes (ver tabla 15), en el 52,3\% de los casos los clientes compran la misma cantidad en tanto que el $24,4 \%$ compran en menor cantidad, confirmando que existe un porcentaje de clientes que están cambiando sus hábitos de compra.

Tabla 15. Cambio cantidad de compra ${ }^{17}$

\begin{tabular}{|l|c|c|c|c|}
\hline & Frequency & Percent & $\begin{array}{c}\text { Valid } \\
\text { Percent }\end{array}$ & $\begin{array}{c}\text { Cumulative } \\
\text { Percent }\end{array}$ \\
\hline $\begin{array}{l}\text { La misma } \\
\text { cantidad }\end{array}$ & 45 & 52,3 & 52,3 & 52,3 \\
\hline $\begin{array}{l}\text { La menor } \\
\text { cantidad }\end{array}$ & 21 & 24,4 & 24,4 & 76,7 \\
\hline $\begin{array}{l}\text { Es mayor } \\
\text { cantidad }\end{array}$ & 20 & 23,3 & 23,3 & 100 \\
\hline Total & 86 & 100 & 100 & \\
\hline
\end{tabular}

Respeto a la frecuencia de compra (ver tabla 16), el $51,2 \%$ de los empresarios consideran que sus clientes van a las empresas a comprar el mismo número de veces, en tanto que el $24,4 \%$ de los mismos consideran que sus clientes van a hacer las compras una menor cantidad de veces, lo cual confirma el cambio de hábitos de compra, manifiesto en la disminución de la frecuencia de compra.
Tabla 16. Frecuencia de compra ${ }^{18}$

\begin{tabular}{|l|c|c|c|c|}
\hline & Frequency & Percent & $\begin{array}{c}\text { Valid } \\
\text { Percent }\end{array}$ & $\begin{array}{c}\text { Cumulative } \\
\text { Percent }\end{array}$ \\
\hline $\begin{array}{l}\text { El mismo número } \\
\text { de veces }\end{array}$ & 44 & 51,2 & 51,2 & 51,2 \\
\hline $\begin{array}{l}\text { Mayor número } \\
\text { veces }\end{array}$ & 21 & 24,4 & 24,4 & 75,6 \\
\hline $\begin{array}{l}\text { Menor número de } \\
\text { veces }\end{array}$ & 21 & 24,4 & 24,4 & 100 \\
\hline Total & 86 & 100 & 100 & \\
\hline
\end{tabular}

En cuanto variación de las referencias de producto compradas por los clientes (ver tabla 17), los empresarios consideran que el $51,2 \%$ de los clientes han variado la mezcla de productos comprados cambiando referencias, mientras que el 31,4\% compra los mismos productos de siempre, lo que es indicativo de la tendencia de un porcentaje importante de clientes a variar y modificar sus hábitos de compra y consumo.

Tabla 17. Variación de la mezcla de productos comprados19

\begin{tabular}{|l|c|c|c|}
\hline & Frequency & Percent & Valid Percent \\
\hline $\begin{array}{l}\text { Los mismos } \\
\text { productos }\end{array}$ & 27 & 31,4 & 31,4 \\
\hline Diferentes productos & 15 & 17,4 & 17,4 \\
\hline $\begin{array}{l}\text { Una mezcla de los } \\
\text { mismos y otros }\end{array}$ & 44 & 51,2 & 51,2 \\
\hline Total & 86 & 100 & 100 \\
\hline
\end{tabular}

Al preguntar a los empresarios sobre las razones para modificar la mezcla de productos ofertados, estos respondieron que el $32,6 \%$ de estos se deben a que los clientes solicitan productos más económicos en tanto que, el $24,4 \%$ solicita mayor surtido de productos (tabla 18).

\footnotetext{
16 Fuente: Elaboración propia a partir de resultados de la investigación. Salida SPSS ${ }^{14}$.

17 Fuente: Elaboración propia a partir de resultados de la investigación. Salida SPSS 14.

18 Fuente: Elaboración propia a partir de resultados de la investigación. Salida SPSS ${ }^{14}$.

19 Fuente: Elaboración propia a partir de resultados de la investigación. Salida SPSS®14.
} 
Tabla 18. Razones de modificación de mezcla de oferta de productos ${ }^{20}$

\begin{tabular}{|l|r|r|r|r|}
\hline & Frequency & Percent & Valid Percent & $\begin{array}{c}\text { Cumulative } \\
\text { Percent }\end{array}$ \\
\hline Productos mas economicos & 28 & 32,6 & 32,6 & 32,6 \\
Mayor surtido de productos & 21 & 24,4 & 24,4 & 57,0 \\
& 8 & 9,3 & 9,3 & 66,3 \\
Presentaciones diversas & 17 & 19,8 & 19,8 & 86,0 \\
Productos con descuento & 12 & 14,0 & 14,0 & 100,0 \\
Productos en combo & 86 & 100,0 & 100,0 & \\
Total & & & \\
\hline
\end{tabular}

Por otra parte, el $36 \%$ de los empresarios considera que su clientela se mantiene, en tanto que el 33,7\% reporta haber perdido clientes (tabla 19).

Tabla 19. Comportamiento de la cantidad de clientes ${ }^{21}$

\begin{tabular}{|l|c|c|c|}
\hline \multicolumn{1}{|c|}{ Cantidad de clientes } & Frecuencia & $\%$ & $\%$ acumulado \\
\hline Definitivamente aumento & 4 & 4,7 & 4,7 \\
\hline Aumento & 9 & 10,5 & 15,1 \\
\hline Se mantiene & 31 & 36 & 51,2 \\
\hline Disminuyo & 29 & 33,7 & 84,9 \\
\hline Definitivamente disminuyo & 13 & 15,1 & 100 \\
\hline Total & 86 & 100 & \\
\hline
\end{tabular}

Las razones principales de la pérdida de clientes de acuerdo con la tabla 20 , son en un $37,2 \%$ de los clientes buscan precios más bajos y el $32,6 \%$ cambian de lugar de vivienda o de trabajo. Esto confirma lo expresado por los jefes de hogar respecto al cambio del sitio de compra por otro donde encuentre precios más bajos.

Tabla 20. Razones de pérdida de clientes 22

\begin{tabular}{|l|c|c|c|c|}
\hline & Frequency & Percent & $\begin{array}{c}\text { Valid } \\
\text { Percent }\end{array}$ & $\begin{array}{c}\text { Cumulative } \\
\text { Percent }\end{array}$ \\
\hline $\begin{array}{l}\text { Cambian de } \\
\text { lugar de vivienda 0 } \\
\text { trabaj0 }\end{array}$ & 28 & 32,6 & 32,6 & 32,6 \\
\hline $\begin{array}{l}\text { Buscan precios } \\
\text { más bajos }\end{array}$ & 32 & 37,2 & 37,2 & 69,8 \\
\hline $\begin{array}{l}\text { Buscan empresas } \\
\text { más cercanas }\end{array}$ & 4 & 4,7 & 4,7 & 74,4 \\
\hline
\end{tabular}

\begin{tabular}{|l|c|c|c|c|}
\hline Buscan crédito & 5 & 5,8 & 5,8 & 80,2 \\
\hline Fallecen & 3 & 3,5 & 3,5 & 83,7 \\
\hline $\begin{array}{l}\text { Buscan mas } \\
\text { amabilidad }\end{array}$ & 2 & 2,3 & 2,3 & 86 \\
\hline Otro & 12 & 14 & 14 & 100 \\
\hline Total & 86 & 100 & 100 & \\
\hline
\end{tabular}

8.4 Descripción del impacto de la variación de la demanda sobre los indicadores de sostenibilidad económica de las empresas estudiadas

Resumiendo los hallazgos, el $36 \%$ de los empresarios encuestados mantienen sus utilidades mientras que para el 33,7\% disminuyen. De las empresas que se han visto afectadas por los cambios de hábitos de compra de los hogares, los clientes que compran menor cantidad a la acostumbrada responde a la causa más representativa presente en el 30,2\% de los casos, El 39,5\% de los empresarios encuestados mantienen sus ventas y el $12,8 \%$ enfrenta un aumento, mientras que el $44,2 \%$ afronta una disminución. Se observa entonces que hay una distribución similar entre mantener y aumentar las ventas, versus disminución de ventas. Finalmente, las ventas aumentan en un $43 \%$ debido a la llegada de nuevos clientes, y en un $15,1 \%$ debido al aumento de la cantidad de productos comprados por los clientes habituales. El $25,6 \%$ de los empresarios reporta una disminución en ventas por baja en los pedidos de los clientes habituales.

\subsection{Correspondencia entre encuesta a hogares y encuesta a empresas}

En la encuesta de hogares, se encontraron entre los cambios en hábitos de compra, el cambio de sitio de compra debido a la búsqueda de menores precios y disminución de la cantidad de compra. En la encuesta de empresas, se encontró que las ventas en empresas localizadas en menor estrato aumentan porque llegan nuevos clientes y en las del estrato habitual hay un menor volumen de compra por cliente. Esto demuestra que hay concordancia entre lo que responden los jefes de hogar y los empresarios.

20 Fuente: Elaboración propia a partir de resultados de la investigación. Salida SPSS ${ }^{2} 14$.

21 Fuente: Elaboración propia a partir de resultados de la investigación. Adaptado de salida SPSS®14.

22 Fuente: Elaboración propia a partir de resultados de la investigación. Salida SPSS®14 


\section{Conclusiones}

De acuerdo con los resultados de la encuesta aplicada a los hogares estratos 3, 4 y 5 de la Localidad de Usaquén, el 97,7\% de estos han enfrentado en los últimos dos años eventos que han disminuido sus ingresos, lo que conduce a un empobrecimiento de los mismos. Este fenómeno se ha visto acompañado del cambio de hábitos de compra de los hogares, evidenciado en la migración hacia establecimientos que ofrecen menores precios, cambios de la mezcla de productos y servicios comprados y eliminación de la compra de algunos productos. Los hogares por tanto, han disminuido el consumo de algunos bienes y servicios, han cambiado la gama de productos comprados, migrado a sitios de compra de menor estrato en busca de precios más favorables y dejado de comprar productos.

Estos cambios de hábitos generan a su vez, un aumento o disminución inesperada de las ventas de las pequeñas y medianas empresas de la localidad, lo cual afecta su sostenibilidad económica, sea mejorándola cuando hay mayores ventas o poniéndola en riesgo, cuando disminuyen las ventas.

Teniendo en cuenta que de las empresas encuestadas el $61 \%$ son unipersonales, el $1 \%$ sociedad en comandita, el 7\% sociedad limitada, el 6\% sociedad anónima y el $7 \%$ sociedad por acciones simplificada SAS, es decir mayoritariamente micros y pequeñas empresas, aunque sensibles a los cambios en la demanda, también cuentan, por su tamaño, con una rápida capacidad de adaptación a los mismos y la evidencia está en las estrategias que están ya aplicando para mantener a los clientes, representadas en un $24 \%$ que aumenta el surtido de productos, un $22 \%$ que mejora las instalaciones para la prestación del servicio y un 20,9\% que mejora la exhibición de productos. Esto demuestra que frente a las variaciones de demanda, estas empresas han desarrollado estrategias adaptativas orientadas a adecuar su oferta a dichos cambios, ampliando su portafolio de oferta y mejorando elementos estructurales como instalaciones de servicio y exhibición de productos, para mantener $y$ atraer clientes.
Ahora bien, como resultado de la triangulación entre los hallazgos de los grupos focales y encuestas aplicadas a los jefes de hogar, se confirmo desde lo cualitativo y lo cuantitativo, que los hogares han cambiado de sitio de compra debido a la búsqueda de precios bajos, disminuido la compra y consumo de algunos productos, cambiado la mezcla de productos habitualmente comprados y enfrentado dificultades económicas mayormente por la pérdida del empleo de uno de los integrantes de la familia.

En cuanto el estudio de empresas, tanto en los hallazgos de las entrevistas a empresarios como los hallazgos de la encuesta, se encontró la solicitud de los clientes de precios más bajos, el cambio en la cantidad y mezcla de compras de los clientes, y la migración de clientes entre estratos.

Al cruzar los resultados de los dos estudios, hogares y empresas, se concluye que efectivamente se están presentado cambios en los hábitos de compra y consumo de los hogares de la muestra, evidenciados en la búsqueda de precios bajos, el cambio de productos y cantidades compradas de estos y la migración hacia establecimientos que ofrecen los precios y el surtido requerido, todo esto motivado por la disminución de ingresos derivada de eventos como pérdida de empleo, cambio a empleo de menor remuneración o baja en las ventas de la propia empresa, lo que es indicativo de un empobrecimiento o lo que es lo mismo pobreza oculta en estos hogares. Pero al mismo tiempo, las empresas están desarrollando estrategias de adaptación para ajustarse a estos nuevos cambios y preservar así su sostenibilidad económica.

Para futuras investigaciones, queda abierto el camino para profundizar en el impacto real que este fenómeno tiene en las cifras financieras de las empresas y negocios del sector y reflexionar sobre cuáles serán las estrategias más adecuadas para la gestión del riesgo que representa la pobreza oculta, para la sostenibilidad de las pequeñas y medianas empresas, tanto de la localidad como en general. 


\section{Referencias}

Bauman, Z. (2007). Vida de consumo. México: Fondo de Cultura Económica.

Cámara de Comercio de Bogotá. (2006). Perfil económico y empresarial Localidad de Usaquén. Bogotá: Cámara de Comercio de Bogotá

Christopher, M. \& Braithwaite, A. (1989). Administración de tiempos rectores. En: Logistics Information Management, 2 (4): 192-197.

Congreso de la República de Colombia. (2000). Ley 590 Promoción del desarrollo de las micro, pequeñas y medianas empresas. Colombia.

DANE (2009). Disponible en: http://www.dane.gov.co/index. php?option $=$ com content\&task $=$ category \&sectionid $=35 \& \mathrm{i}$ $\mathrm{d}=346 \&$ Itemid $=8 \overline{3} 1$

DANE (2010a). Disponible en: http://www.dane.gov.co/index php?option $=$ com content\&task $=$ category \&sectionid $=35 \& \mathrm{i}$ $\mathrm{d}=32 \&$ ltemid $=14 \overline{4}$

DANE (2010b). Ficha metodológica. Disponible en: http://www.dane. gov.co/files/investigaciones/fichas/ficha calidad vida 2008.pdf

Departamento Nacional de Planeación. (2010). Evaluación de la Estratificación socio-económica como instrumento de clasificación de los usuarios y herramienta de asignación de subsidios y contribuciones a los servicios públicos domiciliarios. Serie Evaluación de Políticas Públicas \#10. Disponible en: http://www.dnp.gov.co/PortalWeb/Programas/Sinergia/ EvaluacionesEstrat\%C3\%A9gicas/Evaluacionesdelmpacto/ Estratificaci\%C3\%B3nSocioEcon\%C3\%B3mica/tabid/856/ Default.aspx

Dreze, J. \& Sen, A. (1989). Hunger and public action. Oxford: Clarendon Press.

Guerrien, B. (1998). La Microeconomía. Disponible en: http://www. microeconomia.org/documentos_new/BG-micro.pdf

Lawrence, F. B. (1999). Closing the logisctics loop: a tutorial. En: Production and Inventory Management Journal, 40 (1): 43-51.

Listado de barrios por UPZ. (2009). Disponible en: http://actualidad. vivareal.com.co/2009/03/listado-de-barrios-bogota-por-upz. html
Marsapt, M. (2006). Un forme discrete d pauverete: les personnes logees utilisant les distributions de repas chauds. En: Economie et Statistique No. 391-392: 65-85.

Maslow, A. (1985). El hombre autorrealizado: hacia una psicología del ser. Buenos Aires: Troquel.

Okuda, M., \& Gómez, C. (2005). Métodos en investigación cualitativa, Triangulación. En: Revista colombiana de Psiquiatría XXXIV (001): 118-124.

Pabón, V. (2008) Indicadores de pobreza oculta de la localidad de Teusaquillo. Disponible en: http://www.bibliotecavirtual.estasenteusaquillo.com/pobreza oculta teusaquillo informe.html

Perez, A. (2004). Estrategias feministas de deconstrucción del objeto de estudio de la economía. En: Foro interno: Anuario de Teoría Política: 87-118.

Ravallion, M., Shaohua, C., \& Prem, S. (2007). New evidence on the urbanization of global poverty. En: Policy Research Working Paper Series 4199, The World Bank.

Rowntree, B. (2002). Poverty: A Study of Town Life. International Journal of Epidemiology.

Ruiz, C. (2009). Pobreza relativa y absoluta: el caso de México (1992-2004). En: El trimestre económico , Fondo de Cultura Económica, 0 (301): 67-99.

Secretaria de Planeación. (2009). Disponible en: http://www.sdp. gov.co/www/resources/yap_presentacion sisben 33 33.pdf.

Sen, A. (1976). Poverty: An Ordinal Approach to Measurement. En: Econométrica, 44 (2): 219-231.

Sen, A. (1987). The standard of living. Cambridge: Cambridge University Press.

Serrano, E. D. (1996). Planeación y política social. En: Revista Universidad de Caldas, No. 16.

Uribe, C., Vasquez, S., \& Pardo, C. (2006). Subisidiar y segregar: la política de estratificación y sus efectos en la movilidad social en Bogotá. En: Papel político, Pontificia Universidad Javeriana, 11 (1): 69-94.

www.samuelalcalde.com (2009). Disponible en: http://www.samuelalcalde.com/index.php?option=com_content\&view =article\& $i d=854 \&$ catid $=13 \&$ ltemid $=82$.

Zerda, A. \& Rincón, N. (1998). La pequeña y mediana industria en la encrucijada. Bogotá: Universidad Nacional de Colombia. 